INPLASY

PROTOCOL

To cite: Qiu et al. Retinal blood oxygen saturation and Vascular Endothelial Growth Factor-A in early diabetic retinopathy: a protocol for systematic review and meta-analysis. Inplasy protocol 202040161. doi: 10.37766/inplasy2020.4.0161

Received: 23 April 2020

Published: 23 April 2020

Corresponding author: Qiu Chen

chenqiu1005@cdutcm.edu.cn

Author Affiliation:

Hospital of Chengdu

University of TCM

Support: H2018067

Review Stage at time of this submission: The review has not yet started.

Conflicts of interest: None.

\section{Retinal blood oxygen saturation and Vascular Endothelial Growth Factor-A in early diabetic retinopathy: a protocol for systematic review and meta-analysis}

Qiu, XL1; Wang, X²; Hong, PP3; Liu, M4; Wen, Q5; Yang, XM6; Chen Q7.

Review question / Objective: The purpose of this study is to investigate the association between retinal blood oxygen saturation and vascular endothelial growth factors in patients with early diabetic retinopathy and try to find out the mechanism that the changing of retinal oxygen saturation in patients with diabetic retinopathy.

Information sources: The reference lists of all the included studies, relevant papers and previous systematic reviews will be also hand-searched and analyzed for the identification of additional studies. What's more, we will manually search the database of Chengdu University of Traditional Chinese Medicine Library and our hospital's experts in endocrinology and ophthalmology will be consulted, too.

INPLASY registration number: This protocol was registered with the International Platform of Registered Systematic Review and Meta-Analysis Protocols (INPLASY) on 23 April 2020 and was last updated on 23 April 2020 (registration number INPLASY202040161).

\section{INTRODUCTION}

Review question / Objective: The purpose of this study is to investigate the association between retinal blood oxygen saturation and vascular endothelial growth factors in patients with early diabetic retinopathy and try to find out the mechanism that the changing of retinal oxygen saturation in patients with diabetic retinopathy.

Condition being studied: Diabetic retinopathy (DR) remains the most common microvascular complication of diabetes, both its high prevalence and associated high risk of vision loss lead it to the major global health burden. Despite significant 
research efforts, there still remains much of the underlying pathology not fully understand. Some research has indicated that DR is a direct consequence of hypoxia initiated by hyperglycaemia. Through hypoxiainduced factor alpha (HIF-1a) and expression associated target genes, vascular endothelial growth factor (VEGF) and nitric oxide synthase (NOS), hypoxia is compensated biochemically. High retinal oxygen demand tends to the particular vulnerability of the retina to vascular disease in DR. In the past studies, inner retinal blood flow disturbances is widely assessed as a potential biomarker of DR. However, the results have been variable and even contradictory. Improved methods to figure out the metabolic disturbances associated with DR is essential. Blood oxygen saturation is one of the practical factors of the microcirculation that can reflect metabolic activity. Research on pathological factors related to DR have highlighted the involvement of VEGF-A in conditions. Our study will investigate the association between retinal blood oxygen saturation and VEGF in patients with early diabetic retinopathy and find out the mechanism on it.

\section{METHODS}

Search strategy: [Retinal blood oxygen saturation] AND [Vascular Endothelial Growth Factor-A OR VEGF-A OR Vasculotropin OR VEGF OR Vascular Endothelial Growth Factor OR Vascular Permeability Factor OR Permeability Factor, Vascular OR Glioma-Derived Vascular Endothelial Cell Growth Factor OR Glioma Derived Vascular Endothelial Cell Growth Factor OR GD-VEGF] AND [Diabetic Retinopathy OR Diabetic Retinopathies OR Retinopathies, Diabetic OR Retinopathy, Diabetic].

Participant or population: We will include patients aged $\geq 18$ years with T2DM (according to the WHO criteria) and has been diagnosed with non-proliferative diabetic retinopathy* in observational studies (cross-sectional, case-control and cohort, regardless of sex and ethnicity.
Intervention: Diabetic retinopathy (DR) is an eye condition that can cause vision loss and blindness in people who have diabetes. It affects blood vessels in the retina (the light-sensitive layer of tissue in the back of your eye). In the absence of new vessels, DR is classified as nonproliferative (NPDR) and ranges in severity from mild to severe. In the presence of new vessels, DR is classified as proliferative (PDR). This study will mainly investigate the patients with mild/moderate NPDR: ·Mild NPDR: At least one microaneurysm and definition not met for the other more advanced levels. -Moderate NPDR: H/Ma $\geq$ ETDRS photo 2A in less than 4 quadrants or SE, VB, and IRMA present but milder than other more advanced levels.

Comparator: The controls should be the healthy people who aged $\geq 18$ years without T2DM or any ocular illness that possibly affect ocular circulation.

Study designs to be included: The study will mainly include cross-sectional, casecontrol and both prospective and retrospective cohort studies.

Eligibility criteria: Type of studies crosssectional, case-control and both prospective and retrospective cohortstudies will be included if: They investigate the association between retinal blood oxygen saturation and Vascular Endothelial Growth Factor-A; ·They report data on retinal blood oxygen saturation and Vascular Endothelial Growth Factor; ·They focus on non-proliferative diabetic retinopathy population.

Information sources: The reference lists of all the included studies, relevant papers and previous systematic reviews will be also hand-searched and analyzed for the identification of additional studies. What's more, we will manually search the database of Chengdu University of Traditional Chinese Medicine Library and our hospital's experts in endocrinology and ophthalmology will be consulted, too. 
Main outcome(s): The main outcomes include the values for retinal blood oxygen saturation (Arteriole/ Venule/ AV Difference) and VEGF-A in both NPDRs and controls.

Additional outcome(s): The values for other aqueous humour biomarkers, such as angiopoietin2(Ang2), epidermal growth factor (EGF), hepatocyte growth factor (HGF) and interleukin-8 (IL-8) in both NPDRs and controls.

Quality assessment / Risk of bias analysis: Two reviewers (Qiu and Wang) will independently use different scales to assess the risk of bias based on the type of studies: cross-sectional studies will be assessed by the Agency for Healthcare Research and $Q$ uality (AHRQ) recommended criteria. The criteria include eleven items (table 2), answered by "yes", "no", "unclear"; case-control and cohort studies will use the Newcastle-Ottawa Scale (NOS) which assess the quality of studies with eight questions in three broad categories: (1) patient selection; (2) comparability of study groups; (3) assessment of the outcome. The evaluation will use the semi-quantitative principle of the star system and the highest score is nine stars. Stars of $0-4$ mean low-quality and 5-9 mean high-quality. Any disagreements will be solved by discussion or with arbitrament by the third reviewers Wen or Yang.

Strategy of data synthesis: We will calculate pooled ORs and $95 \% \mathrm{Cls}$. We will assess heterogeneity with the $\mathrm{X} 2$ goodness of fit and 12 statistics. Concerning 12, we will consider Cochrane recommendations. We will think statistically significant a p value $<0.05$ (presence of heterogeneity). In the case of the presence of heterogeneity, we will perform sensitivity analyses and retrogression when possible. When 120.1, the fixed model will be conducted; Otherwise, the random-effects model is about to be employed for meta-analysis. Publication bias will be determined by a funnel plot and we will try to interpret funnel plot asymmetry by Egger's linear regression test if funnel plots are asymmetric.

Subgroup analysis: If there is a sufficient number of studies for each DR, we will investigate potential sources of heterogeneity using metaregression models, and we will perform subgroup analyses by sociodemographic characteristics (eg, age, sex, ethnicity, BMI, life style) and other aqueous humour biomarkers (eg,Ang2, EGF, HGF, IL-8) and by study design(cross-sectional, casecontrol and cohort study).

Sensibility analysis: Sensitivity analysis will be performed to evaluate the quality and stability of meta-analysis results. One analysis solution is to incorporate with random-effect model. On the other hand, we can exclude each included study one by one and re-analysis these dates to pinpoint the trial that induced distinction and finally eliminate it from eligible studies.

Country(ies) involved: China.

Keywords: Diabetes Mellitus, Type 2; retinal disease; blood oxygen saturation; Vascular Endothelial Growth Factor. 